\title{
Radiomic features from MRI distinguish myxomas from myxofibrosarcomas
}

\author{
Teresa Martin-Carreras ${ }^{1}$, Hongming Li ${ }^{1}$, Kumarasen Cooper ${ }^{2}$, Yong Fan ${ }^{1^{*}}$ and Ronnie Sebro ${ }^{1,3,4,5^{*}}$
}

\begin{abstract}
Background: Myxoid tumors pose diagnostic challenges for radiologists and pathologists. All myxoid tumors can be differentiated from each other using fluorescent in-situ hybridization (FISH) or immunohistochemical markers, except for myxomas and myxofibrosarcomas. Myxomas and myxofibrosarcomas are rare tumors. Myxomas are benign and histologically bland, whereas myxofibrosarcomas are malignant and histologically heterogenous. Because of the histological heterogeneity, low grade myxofibrosarcomas may be mistaken for myxomas on core needle biopsies. We evaluated the performance of T1-weighted signal intensity (T1SI), tumor volume, and radiomic features extracted from magnetic resonance imaging (MRI) to differentiate myxomas from myxofibrosarcomas.

Methods: The MRIs of 56 patients (29 with myxomas, 27 with myxofibrosarcomas) were analyzed. We extracted 89 radiomic features. Random forests based classifiers using the T1SI, volume features, and radiomic features were used to differentiate myxomas from myxofibrosarcomas. The classifiers were validated using a leave-one-out cross-validation. The performances of the classifiers were then compared.

Results: Myxomas had lower normalized T1SI than myxofibrosaromas $(p=0.006)$ and the AUC using the T1SI was 0.713. However, the classification model using radiomic features had an AUC of 0.885 (accuracy $=0.839$, sensitivity $=0.852$, specificity $=0.828)$, and outperformed the classification models using T1SI (AUC $=0.713)$ and tumor volume (AUC $=0.838$ ). The classification model using radiomic features was significantly better than the classifier using T1SI values $(p=0.039)$.
\end{abstract}

Conclusions: Myxofibrosarcomas are on average higher in T1-weighted signal intensity than myxomas. Myxofibrosarcomas are larger and have shape differences compared to myxomas. Radiomic features performed best for differentiating myxomas from myxofibrosarcomas compared to T1-weighted signal intensity and tumor volume features.

Keywords: Myxomas, Myxofibrosarcomas, Magnetic resonance imaging, Radiomics, Random forest

\section{Background}

There are several benign and malignant myxoid soft tissue tumors. Benign myxoid tumors include myxomas and angiomyxomas; and malignant myxoid tumors include fibromyxoid sarcomas, extraskeletal myxoid chondrosarcomas, ossifying fibromyxoid tumors, myxoid liposarcomas, myxoinflammatory fibroblastic tumors and myxofibrosarcomas [1]. Each myxoid neoplasms has key chromosomal translocations or immunohistochemical markers that are pathognomic for its diagnosis except for myxomas and myxofibrosarcomas [1-8].

\footnotetext{
*Correspondence: yong.fan@uphs.upenn.edu; Ronnie.sebro@uphs.upenn.edu 'Department of Radiology, University of Pennsylvania, 3400 Spruce Street, Philadelphia, PA 19104, USA

Full list of author information is available at the end of the article
}

Myxofibrosarcomas and myxomas are not associated with any particular translocation or gene expression product, and their diagnoses are based on their histological appearances $[1,8-10]$. Therefore, one of the greatest diagnostic dilemmas for a pathologist lies in differentiating a myxoma from a myxofibrosarcoma, and particularly differentiating a cellular myxoma from some low-grade myxofibrosarcomas [1,8-10]. Differentiation between these two entities is based on morphologic and histologic criteria [1, 8-10]. The challenge for pathologists to differentiate these two entities increases with core needle biopsies because of the heterogeneity of myxofibrosarcomas and because the core biopsy is subject to sampling error [11-13].

(c) The Author(s). 2019 Open Access This article is distributed under the terms of the Creative Commons Attribution 4.0 International License (http://creativecommons.org/licenses/by/4.0/), which permits unrestricted use, distribution, and 
Differentiating myxomas from myxofibrosarcomas is also challenging for radiologists because these lesions have overlapping imaging features - namely they are both hyperintense on T2-weighted magnetic resonance imaging (MRI) sequences, have variable signal intensity on T1-weighted sequences, and both have heterogeneous enhancement patterns [14-18]. Myxomas have been reported to be isointense or hypointense to skeletal muscle using T1-weighted sequences [15]. Myxofibrosarcomas have been reported to be isointense to skeletal muscle on T1-weighted sequences $[18,19]$. The difference is clinically significant because the surgical approach and treatment is different for these two entities. MRIs provide global assessment of the tumor whereas biopsies are limited to certain areas of the tumor; therefore, imaging is likely to be less subject to sampling error.

We hypothesized that quantitative analysis using radiomic feature extraction and classifier model analysis from preoperative MRI studies could predict whether a myxoid tumor is likely to be a myxoma or myxofibrosarcoma over volume-based and MRI signal intensity (SI) value analysis. The purpose of this study was to assess the performance of image intensity information, tumor volume, and radiomic features extracted from MRI for distinguishing myxomas from myxofibrosarcomas.

\section{Methods}

\section{Patients}

This single-center, retrospective case-control study was performed after institutional review board approval, with waiver of the informed consent requirement. The study was performed in compliance with the Health Insurance Portability and Accountability Act (HIPAA). A total of 56 patients who satisfied the inclusion criteria were retrospectively identified. To qualify, patients treated at our institution had to have pre-treatment MRI, and a histologically confirmed (from surgical excision and not from core needle biopsy) diagnosis of myxoma or myxofibrosarcoma between $01 / 01 / 2006$ and 12/31/2017. Patients were excluded from the study if there was no pre-treatment MRI available, if the pre-treatment MRI was motion degraded or did not include a non-contrast enhanced T1-weighted MR imaging sequence, and a fluid-sensitive MR imaging sequence (STIR or T2-weighted sequence). Age and sex was recorded at the time of the histological diagnosis. The maximum size and location of the tumor was determined from evaluation of the pre-treatment MR study.

\section{MRI sequences}

Because these tumors are extremely rare (less than $1 / 100,000)$, patients were sometimes referred to our tertiary center after initial imaging was obtained. The pre-treatment MRIs obtained on these patients were

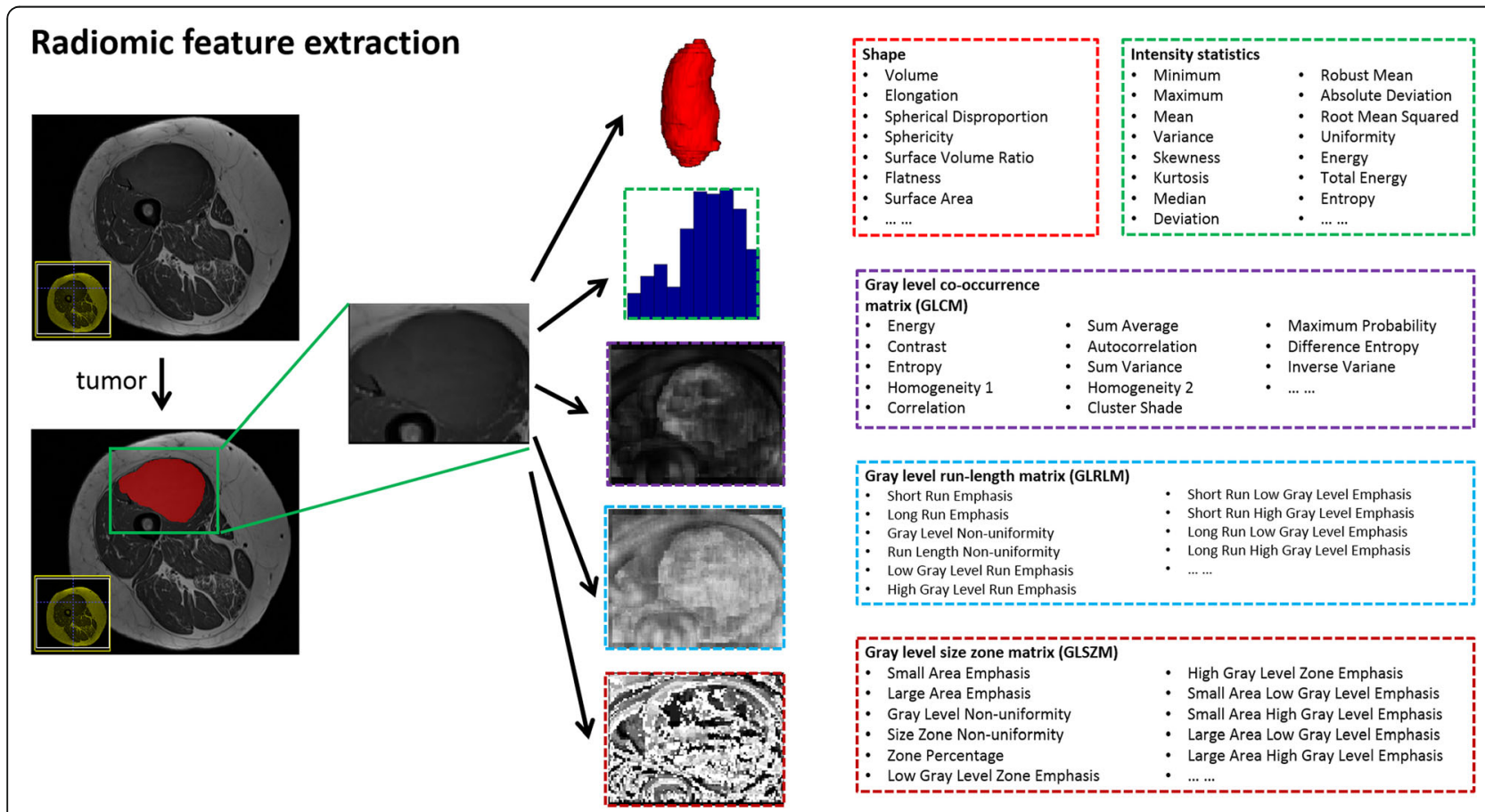

Fig. 1 Extraction of radiomic features from T1 MRI 
usually obtained on a variety of MRI scanners, using several different MR sequences and parameters. We focused on $\mathrm{T} 1$ sequences because this was the most available sequence (all tumor imaging MRI protocols had T1 sequences without fat saturation).

Pre-treatment MRIs were performed using either $0.6 \mathrm{~T}$ (Fonar Corp), 1.2 T (Hitachi Oasis), 1.5 T (Siemens Magnetom Espree; Siemens Avanto; General Electric Medical Systems Optima; General Electric Medical Systems Signa Excite) or $3 \mathrm{~T}$ (Siemens Verio; Siemens Symphony) systems. T1-weighted sequences were as follows: $0.6 \mathrm{~T}$ (repetition time (TR) $414 \mathrm{~ms}$, echo time (TE) $20 \mathrm{~ms}$, slice thickness $5 \mathrm{~mm}$, interslice gap $0 \mathrm{~mm}$, acquisition matrix $1024 \times 200$ ); $1.2 \mathrm{~T}$ (TR 545, TE 12, slice thickness $4 \mathrm{~mm}$, interslice gap $1 \mathrm{~mm}$, acquisition matrix $256 \times 192) ; 1.5 \mathrm{~T}$ (TR 400-600 ms, TE $10-20 \mathrm{~ms}$, slice thickness $3-5 \mathrm{~mm}$, interslice gap $0.5-1 \mathrm{~mm}$ ) and $3 \mathrm{~T}$ (TR $560-700 \mathrm{~ms}$, TE 9-23 ms, slice thickness $4-6 \mathrm{~mm}$, interslice gap 1-1.5 $\mathrm{mm}$, and acquisition matrix $256-320 \times 204-224$ ).

\section{Tissue segmentation}

ITK-SNAP software (open source, http://www.itksnap. org/) was used for manual segmentation of MR images. In all cases, the tumor was segmented on the unenhanced T1-weighted sequences. A reference region-ofinterest (ROI) was drawn in the adjacent normal muscle on unenhanced T1-weighted MR images for image intensity normalization purposes. Adjacent normal muscle had to have no signal abnormality on fluid-sensitive

Table 1 Study demographics

\begin{tabular}{|c|c|c|c|}
\hline & $\begin{array}{l}\text { Myxomas } \\
(N=29)\end{array}$ & $\begin{array}{l}\text { Myxofibrosarcomas } \\
(N=27)\end{array}$ & $P$-value \\
\hline Age in years $(S D)$ & $57.0(12.1)$ & $60.7(15.6)$ & 0.330 \\
\hline No. of women (\%) & $22(75.9 \%)$ & $15(55.6 \%)$ & 0.186 \\
\hline Maximum size $(\mathrm{cm})$ & $3.61(1.3)$ & $10.01(8.0)$ & $<0.001$ \\
\hline Location & & & $0.012^{*}$ \\
\hline Shoulder & 6 & 2 & \\
\hline Arm & 0 & 2 & \\
\hline Forearm & 0 & 3 & \\
\hline Chin & 1 & 0 & \\
\hline Buttock & 5 & 0 & \\
\hline Thigh & 10 & 12 & \\
\hline Leg & 1 & 4 & \\
\hline Foot & 2 & 0 & \\
\hline Pelvis & 3 & 0 & \\
\hline Elbow & 1 & 1 & \\
\hline Knee & 0 & 3 & \\
\hline
\end{tabular}

Clinicodemographic characteristics of patients with myxomas and myxofibrosarcomas

*P-value based on Fisher's exact test sequences (T2-weighted with fat saturation (T2w FS)/ short tau inversion recovery (STIR)) because tumor cells have been found in the peritumoral edema and these tumor cells may affect the T1 signal intensity measured [18]. Reference ROIs in the muscle were drawn to avoid tendons. This reference ROI had to be greater than $35 \mathrm{~mm}^{2}$. Segmentations were done by radiology resident and verified/corrected by a senior musculoskeletal radiologist.

\section{Image intensity normalization}

To adjust for differences in MRI protocols and field strengths the T1-weighted SI was normalized. The normalized intensity map was calculated as $\mathrm{In}=\mathrm{I} / \mathrm{Iref} \times 255$, where I was the original T1 intensity, and Iref was the mean intensity value within the reference ROI. The normalized intensity map was resampled at a spatial resolution of $1 \times 1 \times 1 \mathrm{~mm}^{3}$ before radiomic feature extraction.

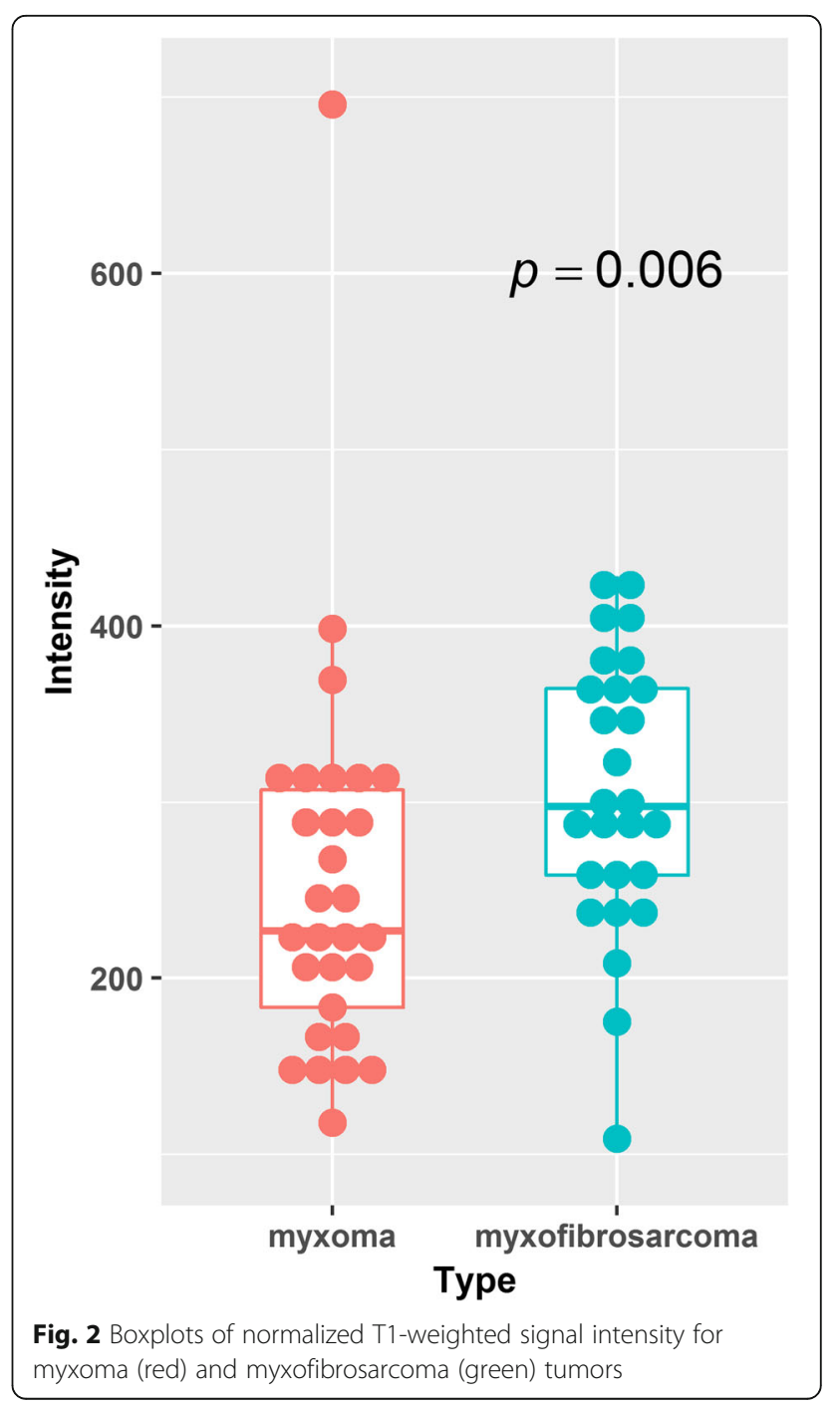




\section{Radiomic feature extraction}

Radiomic features of the tumors were extracted from the normalized intensity map of the $\mathrm{T} 1$ sequence for each patient. In particular, 10 morphologic/volumetric features (volume features) were extracted from each tumor region. Moreover, 79 texture features of the tumor region were extracted from the normalized intensity map, including the first order features, gray level co-occurrence matrix (GLCM) features, gray level size zone matrix (GLSZM) features, and gray level run length matrix (GLRLM) features. All of the features used and their definitions are provided in the Additional file 1: Table S1. In total, 89 radiomic features of the tumor were extracted for each subject. We adopted the fixed bin size strategy for the grey-level discretization with the bin size set to 5 . The feature extraction was carried out in the 3D space and 26-connected neighborhood was adopted. The flowchart for radiomic feature extraction is illustrated in Fig. 1.

\section{Classification}

A random forests based classifier was built upon the radiomic features for distinguishing myxoma from myxofibrosarcomas. The classifiers built upon radiomic features were compared with those based on intensity and volume features in terms of their performance. The number of trees and the minimum leaf size of the random forests classifiers were set to 500 and 3, respectively. The classifiers were validated using a leave-oneout cross-validation. Classification accuracy, sensitivity, specificity, and area under the receiver operating characteristic curve (AUC) were used to evaluate the classification performance. Moreover, the importance for each radiomic feature regarding the prediction was estimated using the out-of-bag permuted predictor delta error. We adopted the implementation for random forests (TreeBagger) in Matlab (R2013a) to train the classifier.

\section{Histopathology}

Histological analysis on the excisional sample was performed by a pathologist. There were 29 patients with myxomas, 5 (18.5\%) patients with grade 1 myxofibrosarcomas, $6(22.2 \%)$ patients with grade 2 myxofibrosarcomas and $16(59.3 \%)$ patients with grade 3 myxofibrosarcomas.

\section{Statistics}

Statistical computing was performed using $\mathrm{R}$ (version 3.4.0) [20]. Variables were compared using WilcoxonRank sum tests for quantitative variables and chisquared tests for qualitative variables. Receiver operating characteristics (ROC) curves and area under the curve

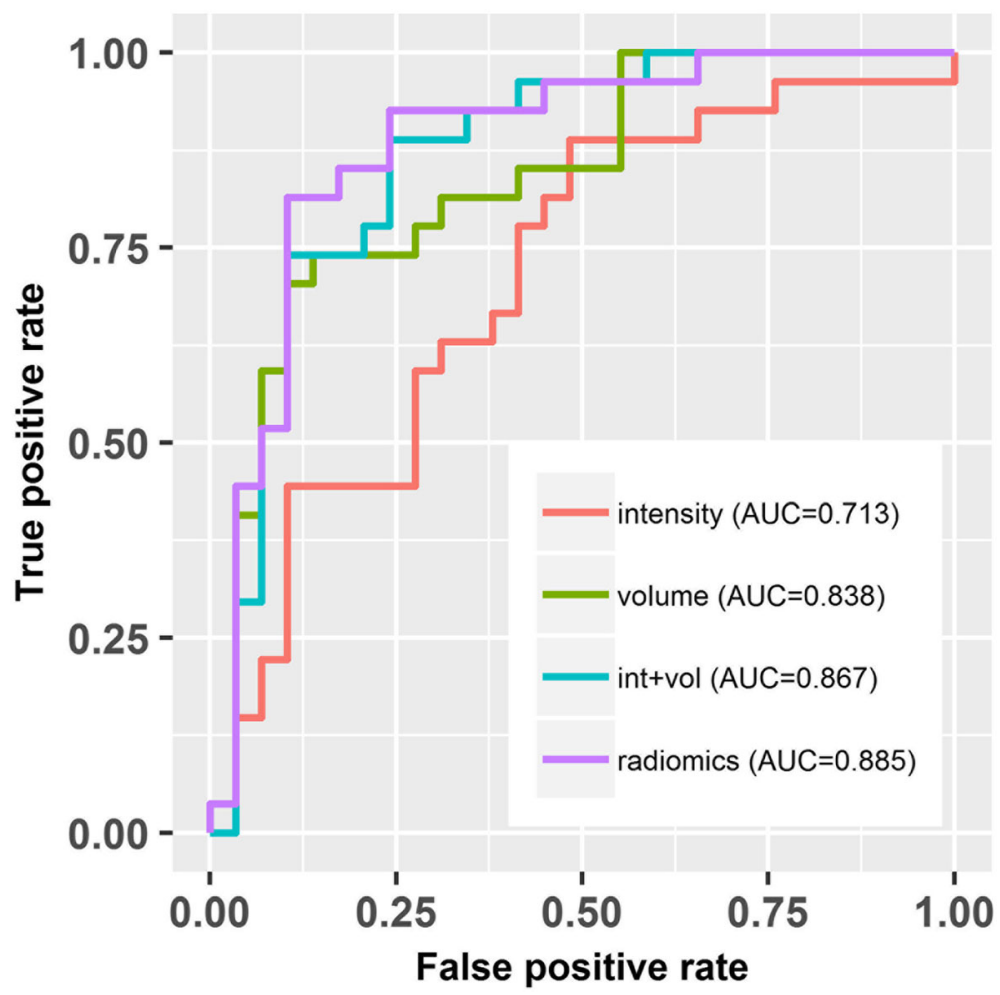

Fig. 3 Receiver-operative characteristic (ROC) curves of classifiers built upon image intensity, volume features, imaging intensity + volume features, and radiomic features 
(AUC) analyses were obtained using the plotROC package. DeLong test (included in the Daim package) was used to compare AUCs of the classifiers built upon different sets of features. All tests were two-sided, and a $P$ value less than 0.05 was considered statistically significant.

\section{Results}

There were a total of 56 patients identified: 29 with myxomas, 27 with myxofibrosarcomas. Subject demographic and clinical variables are shown in Table 1 . There was a higher proportion of female patients with myxomas than myxofibrosarcomas, but the difference was not significantly different ( $p=0.186$ by Pearson's Chi-squared test). None of the patients had a diagnosis of fibrous dysplasia.

As shown in Fig. 2, myxomas had lower normalized T1-weighted signal intensity values than myxofibrosaromas ( $p=0.006$, Wilcoxon rank sum test), and AUC was 0.713 as shown in Fig. 3. Figure 4 shows the T1 SI of the myxofibrosarcomas by tumor grade. There was no substantial difference in the T1 SI between the myxofibrosarcomas by tumor grade (Kruskal-Wallis $p=0.88$ ). The radiomics features for all the subjects were demonstrated in Fig. 5. The classification model built upon radiomic features obtained an AUC of 0.885 (accuracy $=0.839$, sensitivity $=0.852$, specificity $=0.828$ ), which outperformed the classification model built upon the T1SI values ( $p=0.039$, DeLong test), and the classification model built upon volume features (AUC $=0.838, p=$ 0.285 by DeLong test) as shown in Fig. 3 .

To investigate how different features contributed the classification, the top 15 features with high importance regarding classification are demonstrated in Fig. 6. Seven of them were shape-based measures, indicating high association between tumor type and their morphologic properties. This also supported the result that the volume features based classifier had better performance than that based on intensity. The remaining 8 were texture features, and the feature GLSZM_SizeZoneNonUniformity had the highest importance, indicating that texture features were more discriminative, and could provide complementary information to the shape-based features. This suggests that myxofibrosarcomas were more heterogeneous on T1-weighted sequences than myxomas.

\section{Discussion}

The results show that the T1SI of myxofibrosarcomas are on average higher than that of myxomas, however, there was significant T1SI overlap for both lesions. We hypothesize that a more cellular tumor has higher protein content, and would therefore result in increased T1 shortening (higher T1 signal) as our results have shown.

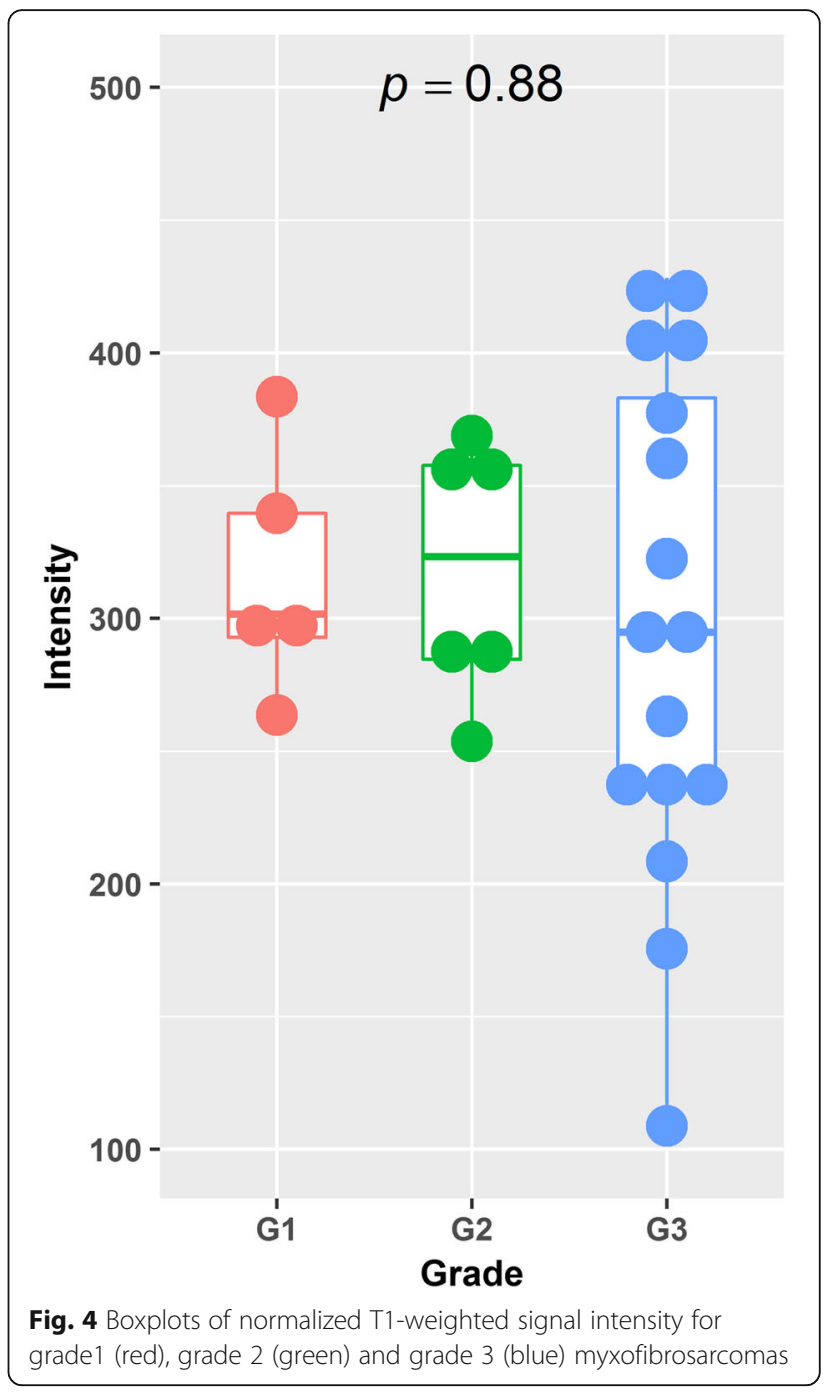

An alternative explanation is that these malignant lesions may contain small foci of hemorrhage. Myxofibrosarcomas also tended to have volumetric features that were slightly different than myxomas. Myxofibrosarcomas have been noted to have a "tail sign" and have a known propensity for spreading along the myofascial planes $[18,21]$. This feature may have been detected as part of the volumetric features.

Radiomic (texture) features were the best for differentiating myxofibrosarcomas from myxomas. Quantitative analysis using a classification model based on radiomic features outperformed the classification models using volume-based and T1SI value analysis. Myxomas tend to be more paucicellular and bland (unless a cellular myxoma), and therefore have less T1 signal heterogeneity. T1-weighted signal heterogeneity of myxofibrosarcomas was greater than that for myxomas, and we speculate that the T1-weighted signal intensity heterogeneity mirrors 

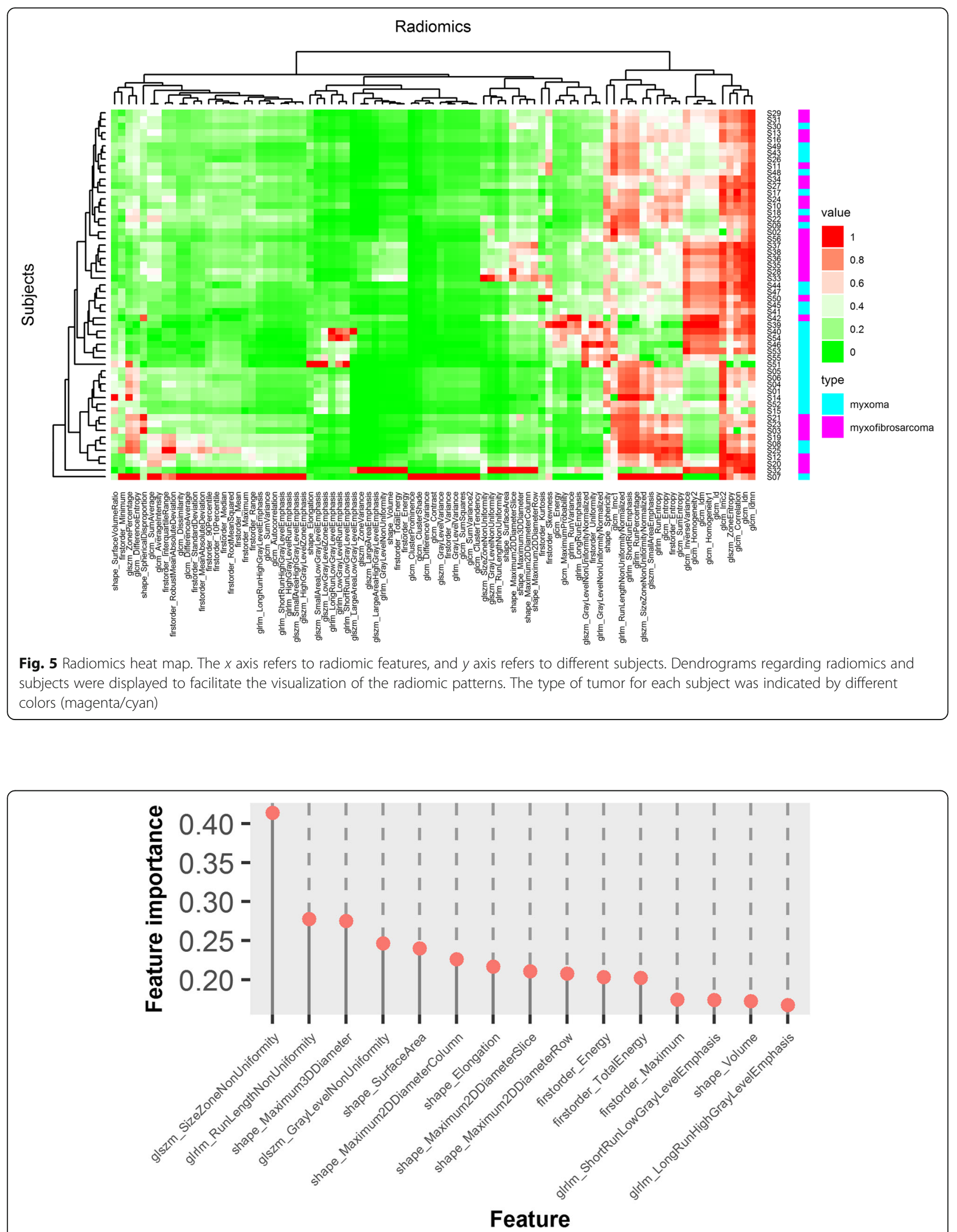

Fig. 6 Top 15 radiomic features with high importance in the random forests based classifier 
the intrinsic histologic tumor heterogeneity seen in myxofibrosarcomas and possibly intratumoral hemorrhage.

Prior reports support our findings. Myxomas have been shown to be hypointense to skeletal muscle on T1weighted sequences $[15,16]$, whereas myxofibrosarcomas have been shown to be more isointense to skeletal muscle on T1-weighted sequences [19]. However, no reports have shown that volumetric and radiomic texture features can be utilized to differentiate myxomas from myxofibrosarcomas from preoperative MRIs.

The results have potential significant clinical implications. Core biopsies are limited by the fact that these lesions are heterogeneous, and the sample cannot entirely represent the lesion's functional and histologic properties [22]. Image-guided core biopsies targeting areas of necrosis in one of the samples may even add to the correct grade specifically in myxofibrosarcoma [23]. Radiomics offers a non-invasive, cost-effective method for assessment of a lesion's entire tumor spatial and temporal heterogeneity [22].

We have shown that MRI image-derived radiomic features can quite accurately differentiate two extremely rare tumor types (myxomas and myxofibrosarcomas) which are challenging for pathologists and radiologists. This is particularly exciting because these tumors are so rare, most radiologists rarely encounter these tumors in daily practice, so most radiologists have limited experience in differentiating these two entities.

Radiomic feature extraction and analysis has been applied broadly to other subspecialties in radiology with successful applications in discerning molecular alterations in tumors, predicting and stratifying tumor response to therapy, and to determine patient prognosis [24-29]. However, literature on musculoskeletal applications of radiomics is scarce. To our knowledge, this is the first study to use radiomic feature extraction and classifier prediction models for this purpose.

The study had some limitations. First, it was retrospective in nature, and subject to ascertainment bias. Myxomas and myxofibrosarcomas are rare tumors (1-4 cases per million people) and this analysis represents one of the largest analyses in the published literature of myxomas and myxofibrosarcomas. Another limitation is that pre-treatment MRIs were obtained using variable parameters and field strengths; however, this was adjusted for by using image intensity normalization. We found that all tumor MRI sequences typically included a T1-weighted sequence, which was why the analysis was restricted to T1-weighted sequences. This makes our findings more broadly applicable to clinical practices which use T1-weighted sequences in their tumor protocols. It is conceivable that there would be additional discriminative information contained in T2 or STIR and post-contrast sequences. We did not analyze the T2/
STIR sequences because these were not always available (some patients had T2-weighted sequences, others had proton density-weighted sequences and others had STIR sequences), which would have left us with a much smaller sample size with limited power due to missing data. Myxofibrosarcomas often have perilesional edema and often have a tail-sign on T2-weighted sequences, which would likely add to the discriminatory ability of the model. Additional research is required to assess whether additional information can be obtained from the T2/STIR sequences to differentiate myxomas from myxofibrosarcomas. We did not analyze the contrast-enhanced T1-weighted sequences because not all patients had contrast enhanced studies and because the time from injection of contrast to imaging was not uniform across patients and we thought this would introduce more noise into the analysis and not be definitive.

\section{Conclusion}

In summary, we have demonstrated that radiomic features from T1-weighted sequences can provide better discriminative information in distinguishing myxoma from myxofibrosarcomas compared to T1-weighted signal intensity values and tumor volume.

\section{Additional file}

Additional file 1: Radiomic features adopted in this study. This file shows how all of the radiomic features used in this study were calculated. (DOCX $28 \mathrm{~kb}$ )

\begin{abstract}
Abbreviations
AUC: Area under the curve; GLCM: Gray level co-occurrence matrix; GLRLM: Gray level run length matrix; GLSZM: Gray level size zone matrix; GLSZM_SizeZoneNonUniformity: Gray level size zone matrix size zone nonuniformity; HIPAA: Health Insurance Portability and Accountability Act; MR: Magnetic resonance; MRI: Magnetic resonance imaging; ROC: Receiver operator characteristic; STIR: Short tau inversion recovery; T1SI: T1-weighted signal intensity
\end{abstract}

\section{Acknowledgements}

The authors' thank the editor and reviewers for their comments which improved the paper.

\section{Ethics approval and consent for participate}

The study was reviewed and approved by the senior authors' institutional review board (IRB). The need for signed informed consent was waived by the University of Pennsylvania Health System IRB (IRB \#823821) because many patients were deceased and others moved without leaving an updated address to contact them.

\section{Authors' contributions}

TMC: Data curation, writing and correcting the manuscript; $\mathrm{HL}$ : statistical analysis, writing and correcting the manuscript, creating figures; KC: Pathology analysis, data curation, writing and correcting the manuscript; YF: writing and correcting the manuscript, statistical analysis; RS: writing and correcting the manuscript, study conceptualization. All authors read and approved the final manuscript. 


\section{Funding}

RS was funded by the Radiology Society of North America Research Scholar and was in part funded by an institutional Sarcoma Pilot Grant. YF and HL were in part funded by National Institutes of Health grants [CA223358, EB022573]. The funding bodies had no influence on the study design, data collection, data analysis, interpretation nor in the writing of the manuscript.

\section{Availability of data and materials}

The datasets used and/or analyzed during the current study are available from the corresponding author on reasonable request.

Image Attribution: All images are our own.

\section{Consent for publication}

The study was reviewed and approved by the senior authors' institutional review board and approved for publication. The need for signed informed consent was waived by the IRB because the results presented do not disclose personal health information (PHI).

\section{Competing interests}

The authors declare that they have no competing interests.

\section{Author details}

'Department of Radiology, University of Pennsylvania, 3400 Spruce Street, Philadelphia, PA 19104, USA. ²Department of Pathology and Laboratory Medicine, University of Pennsylvania, 3400 Spruce Street, Philadelphia, PA 19104, USA. ${ }^{3}$ Department of Orthopedic Surgery, University of Pennsylvania, 3737 Market Street, Philadelphia, PA 19104, USA. ${ }^{4}$ Department of Genetics, University of Pennsylvania, 421 Marie Curie Blvd, Philadelphia, PA 19104, USA ${ }^{5}$ Department of Epidemiology and Biostatistics, University of Pennsylvania, 421 Marie Curie Blvd, Philadelphia, PA 19104, USA.

Received: 7 January 2019 Accepted: 5 August 2019

Published online: 15 August 2019

\section{References}

1. Baheti AD, Tirumani $S H$, Rosenthal MH, Howard SA, Shinagare AB, Ramaiya $\mathrm{NH}$, Jagannathan JP. Myxoid soft-tissue neoplasms: comprehensive update of the taxonomy and MRI features. AJR Am J Roentgenol. 2015;204(2):374-85. https://doi.org/10.2214/AJR.14.12888.

2. Rawlinson, NJ, West WW, Nelson M, Bridge, JA. Aggressive angiomyxoma with $t(12 ; 21)$ and HMGA2 rearrangement: Report of a case and review of the literature. Cancer Genetics and Cytogenetics. 2008; 181 (2): 119-124. https://doi.org/10.1016/j.cancergencyto.2007.11.008. PMC 2396496. PMID 18295664

3. Medeiros F, Erickson-Johnson MR, Keeney GL, Clayton AC, Nascimento AG, Wang $X$, Oliveira AM. Frequency and characterization of HMGA2 and HMGA1 rearrangements in mesenchymal tumours of the lower genital tract. Genes Chromosom Cancer 2007; 46 (11): 981-990. https://doi.org/10.1002/ gcc.20483. PMID 17654722

4. Micci F, Panagopoulos I, Bjerkehagen B, Heim S. Deregulation of HMGA2 in an aggressive angiomyxoma with $\mathrm{t}(11 ; 12)(\mathrm{q} 23 ; \mathrm{q} 15)$. Virchows Arch 2006; 448 (6): 838-842. doi:https://doi.org/10.1007/s00428-006-0186-5. PMID 16568309

5. Nucci MR, Weremowicz S, Neskey DM, Sornberger K, Tallini G, Morton CC, Quade BJ. Chromosomal translocation t $(8 ; 12)$ induces aberrant HMGIC expression in aggressive angiomyxoma of the vulva. Genes Chromosom Cancer 2001; 32 (2): 172-176. doi:https://doi.org/10.1002/gcc.1179. PMID 11550285

6. Yamashita H, Endo K, Takeda C, Teshima R, Osaki M, Yoshida H. Intramuscular myxoma of the buttock mimicking low-grade fibromyxoid sarcoma: diagnostic usefulness of MUC4 expression. Skelet Radiol. 2013; 42(10):1475-9. https://doi.org/10.1007/s00256-013-1641-4 Epub 2013 May 19.

7. Crago AM, Dickson MA. Liposarcoma: multimodality management and future targeted therapies. Surg Oncol Clin N Am. 2016;25(4):761-73.

8. Cates JM, Memoli VA, Gonzalez RS. Cell cycle and apoptosis regulatory proteins, proliferative markers, cell signaling molecules, CD209, and decorin immunoreactivity in low-grade myxofibrosarcoma and myxoma. Virchows Arch. 2015:467(2):211-6. https://doi.org/10.1007/s00428-015-1778-8 Epub 2015 May 5
9. Caraway NP, Staerkel GA, Fanning CV, Varma DG, Pollock RE. Diagnosing intramuscular myxoma by fine-needle aspiration: a multidisciplinary approach. Diagn Cytopathol. 1994;11(3):255-61.

10. Schlenz I, Kuzbari R, Holle J, Worseg A. Soft tissue tumours with difficult to distinguish boundaries. From myxoma to myxofibrosarcoma: a case report. Handchir Mikrochir Plast Chir. 2000;32(2):138-42.

11. Mentzel T, Calonje E, Wadden C, Camplejohn RS, Beham A, Smith MA, Fletcher CD. Myxofibrosarcoma. Clinicopathologic analysis of 75 cases with emphasis on the low-grade variant. Am J Surg Pathol. 1996;20(4):391-405.

12. The ESMO/European Sarcoma Network Working Group. Soft tissue and visceral sarcomas: ESMO Clinical Practice Guidelines for diagnosis, treatment and follow-up. Ann Oncol. 2014;25(Suppl 3):iii102-12.

13. Kasraeian S, Allison DC, Ahlmann ER, Fedenko AN, Menendez LR. A comparison of fine-needle aspiration, core biopsy, and surgical biopsy in the diagnosis of extremity soft tissue masses. Clin Orthop Relat Res. 2010;468(11):2992-3002

14. Bancroft LW, Kransdorf MJ, Menke DM, O'Connor MI, Foster WC. Intramuscular myxoma: characteristic MR imaging features. AJR Am J Roentgenol. 2002;178(5):1255-9.

15. Murphey MD, McRae GA, Fanburg-Smith JC, Temple HT, Levine AM, Aboulafia AJ. Imaging of soft-tissue myxoma with emphasis on $C T$ and MR and comparison of radiologic and pathologic findings. Radiology. 2002;225(1):215-24

16. Abdelwahab IF, Kenan S, Hermann G, Lewis MM, Klein MJ. Intramuscular myxoma: magnetic resonance features. Br J Radiol. 1992;65(774):485-90.

17. Nishio J, Naito M. FDG PET/CT and MR imaging of intramuscular myxoma in the gluteus maximus. World J Surg Oncol. 2012;10:132. https://doi.org/10.11 86/1477-7819-10-132

18. Kaya M, Wada T, Nagoya S, Sasaki M, Matsumura T, Yamaguchi T, Hasegawa T, Yamashita T. MRI and histological evaluation of the infiltrative growth pattern of myxofibrosarcoma. Skelet Radiol. 2008;37(12):1085-90. https://doi. org/10.1007/s00256-008-0542-4 Epub 2008 Jul 16.

19. Petscavage-Thomas JM, Walker EA, Logie Cl, Clarke LE, Duryea DM, Murphey MD. Soft-tissue myxomatous lesions: review of salient imaging features with pathologic comparison. Radiographics. 2014;34(4):964-80. https://doi.org/1 $0.1148 /$ rg.344130110.

20. R Core Team 2015. R: a language and environment for statistical computing 3rd ed. Vienna, Austria: R Foundation for Statistical Computing; 2015. http:// www.R-project.org/.

21. Lefkowitz RA, Landa J, Hwang S, Zabor EC, Moskowitz CS, Agaram NP, Panicek DM. Myxofibrosarcoma: prevalence and diagnostic value of the "tail sign" on magnetic resonance imaging. Skelet Radiol. 2013;42(6):809-18. https://doi.org/10.1007/s00256-012-1563-6 Epub 2013 Jan 15.

22. Sala E, Mema E, Himoto $Y$, Veeraraghavan H, Brenton JD, Snyder A, Weigelt $B$, Vargas HA. Unravelling tumour heterogeneity using next-generation imaging: radiomics, radiogenomics, and habitat imaging. Clin Radiol. 2017:72(1):3-10.

23. Noebauer-Huhmann IM, Amann G, Krssak M, Panotopoulos J, Szomolanyi P, Weber M, Czerny C, Breitenseher M, Grabner G, Bogner W, Nemec S, Dominkus $M$, Funovics $P$, Windhager $R$, Trattnig $S$. Use of diagnostic dynamic contrast-enhanced (DCE)-MRI for targeting of soft tissue tumour biopsies at 3T: preliminary results. Eur Radiol. 2015;25(7):2041-8. https://doi. org/10.1007/s00330-014-3576-0 Epub 2015 Jan 11.

24. Kickingereder P, Götz M, Muschelli J, Wick A, Neuberger U, Shinohara RT, Sill M, Nowosielski M, Schlemmer HP, Radbruch A, Wick W, Bendszus M, MaierHein KH, Bonekamp D. Large-scale Radiomic profiling of recurrent glioblastoma identifies an imaging predictor for stratifying anti-Angiogenic treatment response. Clin Cancer Res. 2016;22(23):5765-71.

25. Shofty B, Artzi M, Ben bashat D, Liberman G, Haim O, Kashanian A, Bokstein F, Blumenthal DT, Ram Z, Shahar T. MRI radiomics analysis of molecular alterations in low-grade gliomas. Int J Comput Assist Radiol Surg. 2018:13(4):563-71.

26. Mcgarry SD, Hurrell SL, Kaczmarowski AL, Cochran EJ, Connelly J, Rand SD, Schmainda KM, LaViolette PS. Magnetic resonance imaging-based Radiomic profiles predict patient prognosis in newly diagnosed glioblastoma before therapy. Tomography. 2016;2(3):223-8.

27. Li H, Galperin-Aizenberg M, Pryma D, Simone CB, Fan Y. Unsupervised machine learning of radiomic features for predicting treatment response and overall survival of early stage non-small cell lung cancer patients treated with stereotactic body radiation therapy. Radiother Oncol. 2018; 129(2):218-26. 
28. Li H, Boimel P, Janopaul-Naylor J, Zhong H, Xiao Y, Ben-Josef E, Fan Y. Deep convolutional neural networks for imaging data based survival analysis of rectal cancer, 2019 IEEE 16th International Symposium on Biomedical

Imaging (ISBI 2019), Venice, Italy, 2019, pp. 846-849. https://doi.org/10.1109/ ISBI.2019.8759301.

29. Men $K$, Geng $H$, Zhong $H$, Fan $Y$, Lin A, Xiao Y. A deep learning model for predicting xerostomia due to radiation therapy for head and neck

squamous cell carcinoma in the RTOG 0522 Clinical Trial. Int J Radiat Oncol Biol Phys. 2019. In press.

\section{Publisher's Note}

Springer Nature remains neutral with regard to jurisdictional claims in published maps and institutional affiliations.

Ready to submit your research? Choose BMC and benefit from:

- fast, convenient online submission

- thorough peer review by experienced researchers in your field

- rapid publication on acceptance

- support for research data, including large and complex data types

- gold Open Access which fosters wider collaboration and increased citations

- maximum visibility for your research: over $100 \mathrm{M}$ website views per year

At BMC, research is always in progress.

Learn more biomedcentral.com/submissions 